\title{
Metatarsophalangeal arthrodesis of the hallux using a minimally invasive technique
}

\author{
Luiz Carlos Ribeiro Lara' $\left({ }^{(\mathbb{D})}\right.$, Lúcio Carlos de Azevedo Torres Filho ${ }^{(}(\mathbb{D})$, Gabriel Lopes de Faria Cervone ${ }^{1(\mathbb{D})}$, Juan Antonio Grajales ${ }^{(\mathbb{D})}$, \\ Glaucia Bordignon ${ }^{1 \mathbb{D}}$, Lara Furtado Lancia' ${ }^{(\mathbb{D})}$, Diego Vitor Braga Santos ${ }^{1}\left(\mathbb{D}\right.$, Igor Marijusckin² ${ }^{\mathbb{D}}$, Douglas Rocha Russo' ${ }^{1 D}$ \\ 1. Hospital Universitário de Taubaté, Taubaté, SP, Brazil. \\ 2. Instituto São Gabriel de Fraturas, Santos, SP, Brazil.
}

\begin{abstract}
Objective: To evaluate the outcomes of the metatarsophalangeal arthrodesis (MTPA) of the hallux using a percutaneous technique.

Methods: The MTPA of the hallux was performed in a total of 27 feet: 20 patients diagnosed with hallux rigidus and 7 with rheumatoid arthritis. The mean postoperative follow-up time was 30.7 months. The results were evaluated using the visual analogue scale (VAS) for pain, the American Orthopaedic Foot \& Ankle Society (AOFAS) forefoot score, and regards to union rate.
\end{abstract}

Results: All 27 patients were operated percutaneously and noticed relief of the pain, with a mean increase of 50.9 points in AOFAS scores and a mean decrease of 7.4 points in the VAS. The mean union time was 10 weeks. There were no cases of nonunion.

Conclusion: Percutaneous first MTP arthrodesis proved effective for treating hallux rigidus and degenerative rheumatic pathologies.

Level of Evidence IV; Therapeutic Studies; Cases Series.

Keywords: Arthrodesis; Metatarsophalangeal joint; Hallux rigidus; Minimally invasive surgical procedures.

\section{Introduction}

Hallux rigidus is the second most common pathology of the first ray of the foot, involving pain and stiffness of the first metatarsophalangeal (MTP) joint ${ }^{(1)}$. Several surgical techniques have been described to relieve symptoms, such as cheilectomy, distal metatarsal osteotomies, MTP arthrodesis, and arthroplasties ${ }^{(2)}$.

MTP arthrodesis was first described in 1883 by Clutton(3), who recommended it for hallux valgus. In 1887, Cotterill(4) proposed using arthrodesis to treat hallux rigidus after having good results with said technique.

Currently, first MTP arthrodesis is considered the gold standard for surgical treatment of hallux rigidus, especially in cases classified as Shurnas and Coughlin(5) grades 3 and 4. Coughlin and Shurnas ${ }^{(6)}$ found good or excellent results in $90 \%$ of the feet they treated with this procedure. The technique can also be used to treat severe deformities, such as those due to rheumatoid arthritis (RA) and post-traumatic sequelae. In search of minimally invasive techniques, several authors have described arthroscopic ${ }^{(7,8)}$ or percutaneous ${ }^{(1,2,9,10)}$ versions of the procedure.
This study aimed to evaluate the results of percutaneous first MTP arthrodesis, including union rate, pain improvement, and patient satisfaction.

\section{Methods}

This retrospective study, which was approved by our institution's ethics committee and registered in Plataforma Brasil (number 36116220.8.0000.5501), was conducted at a university hospital and the private clinic of two of the authors between January 2016 and January 2020. All patients underwent percutaneous MTP arthrodesis following the technique proposed by Bauer et al. ${ }^{(9)}$ in 2010.

The patients underwent pre- and postoperative assessment with the American Orthopaedic Foot and Ankle Society (AOFAS) forefoot score ${ }^{(11)}$, which had been previously translated into Portuguese, and the visual analog scale (VAS) for pain ${ }^{(12)}$, in addition to weight-bearing radiographs in the anteroposterior and lateral projections of the foot. The clinical and radiographic stage of MTP joint arthrosis was classified using the Hattrup and Johnson grading system as modified by Coughlin and Shurnas ${ }^{(6)}$. In the postoperative radiographic
Study performed at the Hospital Municipal Universitário de Taubaté, Taubaté, SP, Brazil.

Correspondence: Luiz Carlos Ribeiro Lara. Av. Itália, 1551, R1 rua 1 casa 666, Jardim das Nações - 12030212, Taubaté-SP , Brazil. E-mail: luizrlara@hotmail.com. Conflicts of Interest: none. Source of funding: none. Date received: July 21, 2021. Date accepted: August 23, 2021. Online: December 20, 2021.
How to cite this article: Lara LCR, Torres Filho

LCA, Cervone GLF, Grajales JA, Bordignon G,

Lancia LF, et al. Metatarsophalangeal arthrodesis

of the hallux using a minimally invasive technique. J Foot Ankle. 2021;15(3):208-12. 
routine, the first MTP joint angle of the hallux was digitally measured on the weight-bearing anteroposterior and lateral view of the foot.

Our sample included patients with moderate or severe arthrosis (grades 3 and 4) of the first MTP joint of the hallux who reported a pain level $>7$ on the VAS.

We excluded patients who had undergone previous surgery on the evaluated foot, had MTP arthrosis due to traumatic sequelae or had less than 6 months of postoperative follow-up.

Initially, the data were analyzed descriptively. Categorical variables were expressed as absolute and relative frequencies, while numerical variables were expressed as summary measures (mean, median, minimum, maximum, and standard deviation).

The distributions of characteristics and means between the groups were compared using Fisher's exact test and Student's $t$-test for independent samples, respectively. The mean AOFAS and VAS scores between the time points were compared using Student's $t$-test for paired samples. The mean AOFAS scores between the time points according to the group diagnose were compared using a mixed linear model. The mixed linear model incorporated the effect of each patient in the form of a random effect, considering a possible dependence between observations in the same individual.

Normal distribution of the data was assumed in both Student's $t$-test and the mixed linear model, which was verified with the Kolmogorov-Smirnov test. A significance level of $5 \%$ was used for all tests. The statistical analyses were performed in SPSS 20.0 and Stata 12.

\section{Material and patient positioning}

The procedures were performed by a team of five orthopedic surgeons specialized in Foot and Ankle Surgery. While preparing for the procedure, the patient was positioned in horizontal dorsal decubitus with the feet off of the operating table, under locoregional anesthesia(13). No tourniquets were used. A MIS 64 blade (BD Beaver Blades, Becton Dickinson, 1 Becton Drive, Franklin Lakes, NJ), a $3.1 \mathrm{~mm}$ wedge burr, and a long Shannon burr were used to perform the technique.

\section{Surgical approach}

The initial approach was at the medial edge of the MTP joint, guided by a gauge needle inserted in the joint space. Next, a $0.5 \mathrm{~cm}$ incision was made with the MIS 64 blade. A second lateral access to the MTP joint was made using the same technique. In patients with a prominent dorsal exostosis, a third, more dorsal incision was used for adequate resection (Figure 1).

\section{Exostectomy and joint preparation}

The exostosis was thinned using a $3.1 \mathrm{~mm}$ wedge burr through a dorsomedial surgical access point. The resection was guided by radioscopy until a flat surface was obtained on the metatarsal head.
The joint was prepared with a long Shannon burr, seeking to remove the entire cartilaginous surface (Figure 2 ). With a rasp, joint fragments were removed.

\section{Arthrodesis and fixation}

The MTP joint was positioned for arthrodesis under fluoroscopy and with a radiolucent platform to imitate plantar foot support. After obtaining the ideal position of $15^{\circ}$ dorsiflexion in relation to the platform and $5-10^{\circ}$ of valgus in the MTP joint, provisional fixation of the MTP joint was performed using two $1.5 \mathrm{~mm}$ Kirschner wires crossed in a medial and lateral configuration. Fixation was then performed with self-compressing $2.7 \mathrm{~mm}$ Herbert-type cannulated screws (Figure 3).

\section{Postoperative care}

Walking with hard-soled sandals was allowed after the first postoperative day. The patients returned to our office in each of the first 4 postoperative weeks to change the dressing and, in the subsequent 4 weeks, they changed the dressing

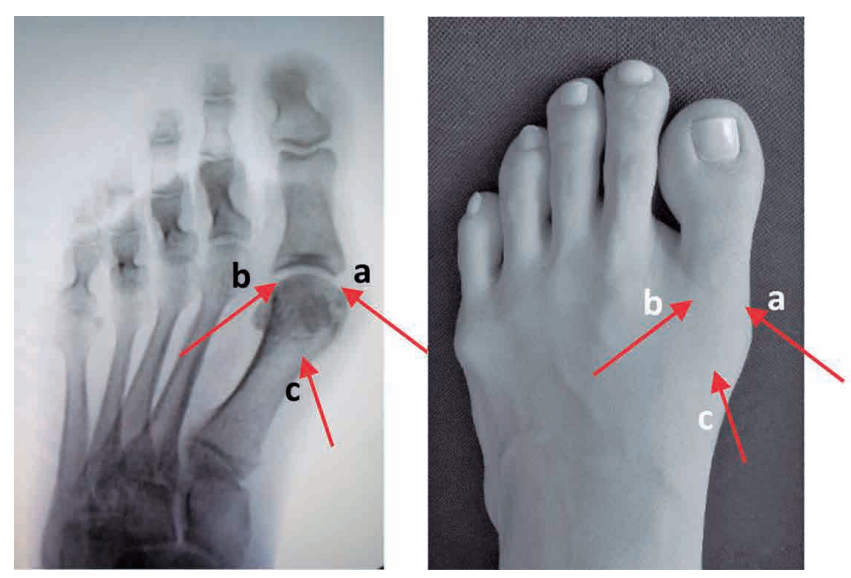

Figure 1. Radioscopy-guided access routes $(A)$ and their skin incision sites (B): A - medial route; $B$ - lateral route for joint preparation; $\mathrm{C}$ - accessory pathway for dorsal thinning.
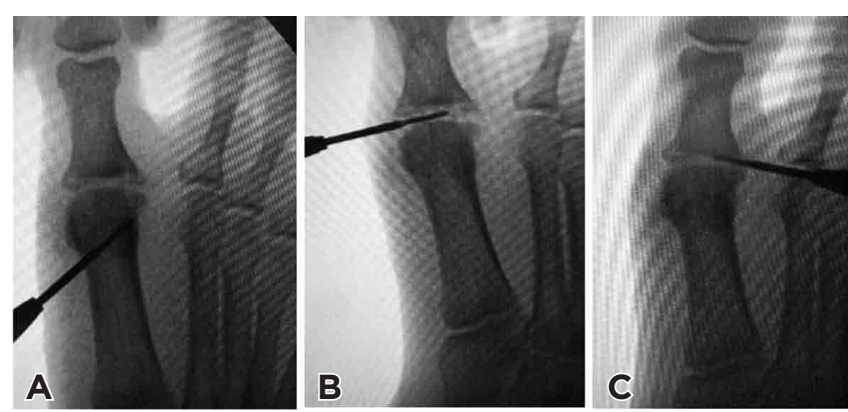

Figure 2. A) Intraoperative radioscopy illustrating the technique for removing dorsal osteophytes and B) removing articular cartilage and C) preparing the surfaces for arthrodesis. 

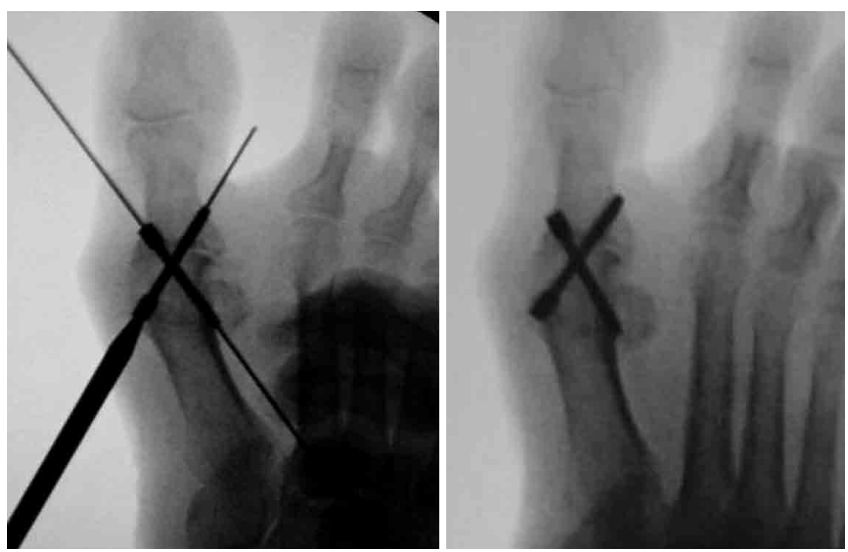

Figure 3. Intraoperative radioscopic image demonstrating the fixation technique with self-compressing Herbert screws.

at home. Radiographic control was requested in the 1st, 4th, 8 th, and 12th postoperative weeks. In cases of delayed union, new monthly radiographs were requested until the union was radiographically proven.

\section{Results}

We analyzed data from 27 patients with a mean age of 57.4 years (SD, 8.5 years; range 38 to 73 years). Twenty of these patients $(74.1 \%)$ were diagnosed with hallux rigidus, and the other 7 were diagnosed with RA.

Overall, $63 \%$ (17) of the patients were female. The mean follow-up time was 30.7 months (SD, 8.7) and the mean union time was 2.8 months (SD, 1.2), ranging from 4 to 24 weeks (or 1 to 6 months, as shown in Table 1). The patients' mean VAS pain score decreased by 8.4 points (SD, 1.3) between the pre- and postoperative periods.

Table 2 shows the differences in mean AOFAS $(p<0.001)$ and VAS $(p<0.001)$ scores between the time points. There was a mean increase of 50.9 points (SD, 13.5) in AOFAS scores and a mean reduction of 7.4 points (SD, 1.5 points) in VAS scores. In both groups, mean increases were observed between the first and second AOFAS applications $(p<0.001)$ : 62.1 points in the RA group, and 47.0 points in the hallux rigidus group. However, the mean increase in the RA group was 15.2 points higher than that of the hallux rigidus group (95\% Cl: 5.3 - 25.1).

Among the complications, there was a case of intraoperative guidewire breakage. The patient initially complained of grade 3 pain on the VAS scale in the lateral and dorsal region of the MTP, but it evolved to spontaneous resolution. Thus, there was no need to remove the wire, which remained in place as a lost synthesis. In 2 feet, screw removal was necessary due to complaints of discomfort. The arthrodesis union was delayed in 1 patient, including signs of bone callus only after 24 weeks postoperatively. There were no cases of malunion, infection, deep vein thrombosis, or severe pain.

\section{Discussion}

Two pathologies were found to cause alterations in the MTP joint in this sample: rheumatoid arthritis and hallux rigidus. Most of the cases were due to hallux rigidus (20 feet), with only 7 patients in the RA group. This finding may be because hallux rigidus is the main degenerative pathology of the first MTP joint, which typically causes pain, limited mobility, and periarticular exostosis with subsequent deformation of the joint itself ${ }^{(14-16)}$. Even with radiographs indicating osteodegenerative changes in the joint, we believe that pain is an important clinical parameter for indicating MTP arthrodesis, since this is the main patient complaint, and pain improvement helps quantify postoperative results ${ }^{(17,18)}$. Although there is no gold standard for analyzing the results, the AOFAS forefoot scale provided us with enough information to compare the results of this surgical technique.

Although other studies have shown good or excellent results for first MTP arthrodesis through open ${ }^{(4,17-21)}$ and arthroscopic ${ }^{(7,8)}$ surgery, percutaneous surgery has certain advantages over these techniques, since it is less invasive and involves little aggression to the soft tissues, in addition to being performed with locoregional anesthesia and allowing early ambulation ${ }^{(2,11)}$

Unlike Bauer et al. ${ }^{(9)}$, we did not use a tourniquet on the calf to keep the member exsanguinated, since the blood flowing through the incision helps cool the burr, preventing bone and soft tissue burns.

With the percutaneous technique, the joint can be prepared with a "flat to flat" interface ${ }^{(1,9,10)}$ whose results are very similar to the convex-concave joint preparations of open surgery $(1,2,17)$.

Another divergent point in the literature is whether or not to maintain the bone debris produced by the exostectomy ${ }^{(2)}$. We understand that it should be maintained and that it helps in the bone callus formation process. We agree with Bauer ${ }^{(10)}$ and Nogueira et al. ${ }^{(18)}$ that bone resection must be performed carefully to avoid shortening the first ray and poor positioning.

Regarding fixation methods, according to the literature, using plates and screws specifically designed for MTP arthrodesis and fixation with two crossed screws are equally rigid and strong ${ }^{(19,20)}$. Thus, percutaneous screw fixation can obtain excellent results. Coughlin and Shurnas followed up more than 200 patients who received fixation with 2 percutaneous screws, finding good results in more than $90 \%$ of cases, in addition to short surgery time, quick recovery, and few surgical complications $^{(6)}$

The mean union time in our sample was 2.8 months, slightly higher than that of Nogueira et al. ${ }^{(18)}$, who reported bone callus formation 2 months after surgery. Regarding possible nonunions, Bauer ${ }^{(10)}$ reported that radiological nonunion occurred in $10 \%$ of his patients, although they were asymptomatic. Union occurred in all of the feet in our sample. Callus formation was delayed in only one foot, although complete MTP fusion had occurred 6 months postoperatively. 
Table 1. Patient characteristics

\begin{tabular}{|c|c|c|c|c|}
\hline & Total $(\mathrm{N}=27)$ & $\mathrm{RA}(\mathrm{N}=7)$ & $\mathrm{HR}(\mathrm{N}=20)$ & $\mathbf{p}$ \\
\hline Sex & & & & $0.678^{a}$ \\
\hline Female & $17(63.0)$ & $5(71.4)$ & $12(60.0)$ & \\
\hline Male & $10(37.0)$ & $2(28.6)$ & $8(40.0)$ & \\
\hline Age (years) & & & & 0.195 \\
\hline Mean $\pm S D$ & $57.4 \pm 8.5$ & $61.0 \pm 6.2$ & $56.1 \pm 9.0$ & \\
\hline Median (Minimum - Maximum) & $58(38-73)$ & $62(51-68)$ & $56.5(38-73)$ & \\
\hline Follow-up (months) & & & & 0.420 \\
\hline Mean \pm SD & $30.7 \pm 8.7$ & $33.0 \pm 11.6$ & $29.9 \pm 7.6$ & \\
\hline Median (Minimum - Maximum) & $28(22-52)$ & $30(22-52)$ & $26.5(22-43)$ & \\
\hline Preoperative AOFAS & & & & 0.020 \\
\hline Mean \pm SD & $29.3 \pm 11.7$ & $20.7 \pm 12.7$ & $32.4 \pm 9.9$ & \\
\hline Median (Minimum - Maximum) & $30(5-45)$ & $15(5-40)$ & $35(15-45)$ & \\
\hline Preoperative VAS & & & & 0.711 \\
\hline Mean \pm SD & $8.4 \pm 1.3$ & $8.3 \pm 1.4$ & $8.5 \pm 1.3$ & \\
\hline Median (Minimum - Maximum) & $9(6-10)$ & $8(7-10)$ & $9(6-10)$ & \\
\hline UT (months) & & & & 0.257 \\
\hline Mean \pm SD & $2.8 \pm 1.2$ & $2.4 \pm 0.5$ & $3.0 \pm 1.3$ & \\
\hline Median (Minimum - Maximum) & $2.5(1-6)$ & $2(2-3)$ & $2.8(1-6)$ & \\
\hline
\end{tabular}

AOFAS: American Orthopedic Foot and Ankle Society Forefoot Score. HR: hallux rigidus. RA: rheumatoid arthritis; SD: standard deviation; UT: union time; VAS: Visual analog scale for pain.

Table 2. Measures - summary of AOFAS and VAS scores according to assessment time

\begin{tabular}{lcccc} 
& \multicolumn{2}{c}{ Time of evaluation } & Post-Pre & p \\
\cline { 2 - 3 } & Pre & Post & & \\
\hline AOFAS & $29.3 \pm 11.7$ & $80.2 \pm 6.6$ & $50.9 \pm 13.5$ & $<0.001$ \\
VAS & $8.4 \pm 1.3$ & $1.0 \pm 1.1$ & $-7.4 \pm 1.5$ & $<0.001$ \\
\hline
\end{tabular}

p: descriptive
Mean \pm SD.

AOFAS: American Orthopedic Foot and Ankle Society Forefoot Score; VAS: Visual analog scale for pain.

We agree with Bauer(10) that the main strategies for a successful first MTP arthrodesis are decortication of the entire articular surface, fixation with 2 cannulated or self-compressing screws, correct positioning of the MTP joint for arthrodesis, and avoiding excessive bone decortication in patients with extensive osteochondral lesions or severe osteoporosis. Following these guidelines, firm and stable fixation is guaranteed ${ }^{(9)}$. We believe that no cases of malunion (or union in a non-ideal position) occurred because we maintained the angle described in the surgical technique.
As study limitations, we point out that the same team of surgeons performed both the surgeries and the pre- and postoperative assessments, which could lead to performance bias. Since this was a retrospective study to assess the results of a single surgical technique, there was no control group.

The percutaneous technique results in less aggression to soft tissues and allows ambulation in the immediate postoperative period ${ }^{(1,2,9,10)}$. However, the surgeon's experience level is also important, since the learning curve for this technique is long and can lead to unsatisfactory results if performed poorly. Since no large-scale randomized comparative studies have demonstrated the superiority of any one technique, we conclude that both open and minimally invasive procedures are useful tools in the arsenal of orthopedists and podiatrists.

\section{Conclusions}

Percutaneous arthrodesis proved to be an effective treatment for hallux rigidus and RA in the first MTP joint, with an excellent rate of union and patient satisfaction. Both AOFAS and VAS pain scores improved significantly in the postoperative period. 
Authors' contributions: Each author contributed individually and significantly to the development of this article: LCRL *(http://orcid.org/OOOO-OOO31158-2643) Conceived and planned the activities that led to the study, performed the surgeries, data collection, clinical examination, approved the final version; LCATF *(https://orcid.org/0000-0002-0778-2506) Conceived and planned the activities that led to the study, performed the surgeries, data collection, clinical examination; GLFC *(https://orcid.org/0000-0001-5470-8379) Conceived and planned the activities that led to the study, performed the surgeries, data collection, clinical examination; JAG *(https://orcid.org/0000-0003-4652-4400) Conceived and planned the activities that led to the study, performed the surgeries, data collection, clinical examination; GB *(https://orcid.org/0000-0001-5273-4303) Wrote the article, interpreted the results of the study, participated in the review process, statistical analysis, bibliographic review, survey of medical records, formatting of the article; LFL *(https:// orcid.org/0000-0003-1048-7134) Wrote the article, interpreted the results of the study, participated in the review process, statistical analysis, bibliographic review, survey of medical records, formatting of the article; DVBS *(https://orcid.org/0000-0001-6988-1609) Wrote the article, interpreted the results of the study, participated in the review process, statistical analysis, bibliographic review, survey of medical records, formatting of the article; IM *(https://orcid. org/0000-0002-1733-3747) Conceived and planned the activities that led to the study, performed the surgeries, data collection, clinical examination; DRR *(https://orcid.org/0000-0001-9594-2006) Conceived and planned the activities that led to the study, performed the surgeries, data collection, clinical examination. All authors read and approved the final manuscript. * ORCID (Open Researcher and Contributor ID) iD).

\section{References}

1. Sott AH. Minimally Invasive Arthrodesis of 1st Metatarsophalangeal Joint for Hallux Rigidus. Foot Ankle Clin. 2016;21(3):567-76.

2. Fanous RN, Ridgers S, Sott AH. Minimally invasive arthrodesis of the first metatarsophalangeal joint for hallux rigidus. Foot Ankle Surg. 2014;20(3):170-3.

3. Clutton H. The treatment of hallux valgus. St Thomas Hosp Rep 1894;22:1-12.

4. Cotterill JM. Stiffness of the great toe in adolescents. Br Med J. 1887;1(1378):1158.

5. Shurnas PS, Coughlin MJ. Arthritic Conditions of the Foot. Coughlin MJ, Mann RA, Saltzman CL. Surgery of the Foot and Ankle. $8^{\text {th }}$ ed. Philadelphia: Mosby Elsevier; 2007. p. 837-51.

6. Coughlin MJ, Shurnas PS. Hallux rigidus: demographics, etiology, and radiographic assessment. Foot Ankle Int. 2003;24(10):731-43.

7. Carro LP, Vallina BB. Arthroscopic-assisted first metatarsophalangeal joint arthrodesis. Arthroscopy. 1999;15(2):215-7.

8. Walter R, Perera A. Open, Arthroscopic, and Percutaneous Cheilectomy for Hallux Rigidus. Foot Ankle Clin. 2015;20(3):421-31.

9. Bauer T, Lortat-Jacob A, Hardy P. First metatarsophalangeal joint percutaneous arthrodesis. Orthop Traumatol Surg Res. 2010; 96(5):567-73.

10. Bauer T. Percutaneous First Metatarsophalangeal Joint Fusion. Open Orthop J. 2017;11:724-31.

11. Johnson C. Measuring Pain. Visual Analog Scale Versus Numeric Pain Scale: What is the Difference? J Chiropr Med. 2005;4(1):43-4.

12. Kitaoka HB, Alexander IJ, Adelaar RS, Nunley JA, Myerson MS, Sanders M. Clinical rating systems for the ankle-hindfoot, midfoot, hallux, and lesser toes. Foot Ankle Int. 1994;15(7):349-53.
13. Torres Filho LCA, Lara LCR, Cervone GLF, Figueiredo RN, Lancia LF. 4-in-1 and 5-in-1 blocks in percutaneous forefoot surgery. J Foot Ankle. 2020;14(1):79-83.

14. Hogan MV, Mani SB, Chan JY, Do H, Deland JT, Ellis SJ. Validation of the Foot and Ankle Outcome Score for Hallux Rigidus. HSS J. 2016;12(1):44-50.

15. Lucas DE, Hunt KJ. Hallux Rigidus: Relevant Anatomy and Pathophysiology. Foot Ankle Clin. 2015;20(3):381-9.

16. Hattrup SJ, Johnson KA. Subjective results of hallux rigidus following treatment with cheilectomy. Clin Orthop Relat Res. 1988; (226):182-91.

17. Coughlin MJ, Shurnas PS. Hallux rigidus. Grading and longterm results of operative treatment. J Bone Joint Surg Am. 2003;85(11):2072-88

18. Nogueira VB, Pereira Filho MV, Mattos e Dinato MC, Freitas MTP, Pagnano RG. Treatment of hallux rigidus with percutaneous arthrodesis: a case series. Sci J Foot Ankle. 2018;12(2):90-5.

19. Harris E, Moroney P, Tourné Y. Arthrodesis of the first metatarsophalangeal joint-A biomechanical comparison of four fixation techniques. Foot Ankle Surg. 2017;23(4):268-74.

20. Korim MT, Mahadevan D, Ghosh A, Mangwani J. Effect of joint pathology, surface preparation and fixation methods on union frequency after first metatarsophalangeal joint arthrodesis: A systematic review of the English literature. Foot Ankle Surg. 2017;23(3):189-94.

21. Curtis MJ, Myerson M, Jinnah HR, Cox QG, Alexander I. Arthrodesis of the first metatarsophalangeal joint: a biomechanical study of internal fixation techniques. Foot Ankle. 1993;14(7):395-9. 